\title{
Síndrome da reperfusão encefálica. Hipóteses fisiopatológicas, bioquímicas e hemodinâmicas do fenômeno da hipoperfusão pós-hiperemia
}

\author{
Marcos Augusto Stávale Joaquim¹, Gustavo Cartaxo Patriota', André de Macedo Bianco
}

Instituto de Neurociências, São Paulo, SP.

Serviço de Neurocirurgia do Hospital Nove de Julho, São Paulo, SP.

\section{RESUMO}

O objetivo deste texto é o de estruturar e sistematizar novas hipóteses fisiopatológicas sobre as modificações hemodinâmicas do cérebro submetido à isquemia e à reperfusão. São propostas e analisadas hipóteses bioquímicas e hemodinâmicas do fenômeno da hipoperfusão pós-hiperemia.

\author{
PALAVRAS-CHAVE \\ Isquemia cerebral. Síndrome da reperfusão.
}

\section{ABSTRACT}

Reperfusion syndrome. Pathopysiological hypothesis

The aim of this publication is to elaborate and discuss a new pathophysiological hypothesis about the haemodinamic modifications into the brain that occur after the reperfusion. Biochemical and haemodinamic hypothesis are proposed and analyzed, concerning the hipoperfusion following the hiperemia phenomenon.

\section{KEY WORDS}

Brain ischemia. Reperfusion injury.

\section{Introdução}

O fenômeno da hipoperfusão pós-hiperemia é uma isquemia tardia que ocorre após uma fase de hiperemia.

A reperfusão do tecido isquêmico pode ocasionar três tipos de fenômenos. No primeiro, a hiperemia reativa transitória ocorre se a isquemia foi leve, pelo acúmulo de substâncias vasodilatadoras teciduais que incluem o hidrogênio iônico e a adenosina. É um fenômeno fisiológico autolimitado e não eleva a pressão intracraniana (PIC) mesmo que grandes áreas sejam acometidas. ${ }^{41}$ No segundo, o fenômeno da não reperfusão (no-reflow) ocorre se a isquemia foi intensa e houver edema e formação de vilosidades no endotélio, tromboses e oclusão da microcirculação. No terceiro, uma situação intermediária, o fenômeno da hipoperfusão pós-hiperemia ocorre após isquemias moderadas. ${ }^{43,44,47,52,59,64-66,71}$

Diversas publicações diferenciam os inchaços cerebrais hipervolêmicos (swelling) em hiperêmicos e oligoêmicos. Embora ambos devam-se ao aumento do volume sanguíneo encefálico (VSE), associam-se, respectivamente, a fluxo sanguíneo encefálico (FSE) e pressão de perfusão encefálica (PPE) altos no primeiro tipo e baixos no segundo tipo. Tais situações são fases evolutivas de um mesmo processo hemodinâmico que ocorre na hipertensão intracraniana (HIC) progressiva e são particularmente relacionados à síndrome da reperfusão. ${ }^{37-40,43,44,48,49,51,54,71}$ 


\section{Bases fisiopatológicas}

A isquemia gera ácido láctico tecidual. O hidrogênio iônico ativa as cascatas vasodilatadora e bioquímica no tecido encefálico em sofrimento. ${ }^{66,67,71}$

A cascata vasodilatadora diz respeito a uma hipervolemia encefálica progressiva. Como o $\mathrm{pH}$ periarteriolar é o principal controlador da capacidade contrátil da arteríola pré-capilar, a acidose causa vasodilatação do sistema de resistência arteriolar (também chamado de mecanismo de autorregulação da circulação cerebral MARCC) e preenchimento secundário do sistema vascular de capacitância (microcirculação, vênulas e veias que contém $70 \%$ do VSE). Há aumento progressivo do VSE, que pode gerar inchaço cerebral a ponto de produzir HIC. Se esta ocorrer, há diminuição secundária da PPE e mais isquemia, que provoca mais vasodilatação, aumento adicional do VSE e da HIC e queda subsequente da PPE. Desencadeia-se um círculo vicioso vasodilatador que leva a HIC refratária ao tratamento. Esse processo desenvolve a fase ascendente exponencial da PIC na curva de Langfitt. ${ }^{13-15,25,28,30-33,35,36,46,48,49,51,52,55,56,66-69}$

A cascata bioquímica diz respeito à série de alterações metabólicas e iônicas que desencadeiam a morte celular. Tais alterações também são desencadeadas pela acidose tecidual e parte delas foi conhecida estudando-se experimentalmente a síndrome da reperfusão, que é uma maneira de se estudarem isquemias moderadas com fornecimento parcial de oxigênio aos tecidos, pois produzem as mesmas sequências de alterações bioquímicas. ${ }^{19,22,26,45,53,55,67,69}$

\section{Hipoperfusão pós-hiperemia sob o ponto de vista hemodinâmico: primeira hipótese}

Na relação entre reperfusão e hemodinâmica intracraniana, o processo de inchaço acentua-se após a devolução da PPE a um leito vascular dilatado pela acidose secundária à isquemia que ocorreu primariamente. Tal isquemia, geralmente, deve-se à elevação inicial da PIC por hematomas espontâneos ou traumáticos que alcançam um volume suficiente para diminuir a PPE e depois são drenados (a drenagem imediata é obrigatória no tratamento se há elevação da PIC). Tal reperfusão também ocorre após a parada cardiorrespiratória reanimada. ${ }^{13-16}$

A devolução da PPE à microcirculação dilatada causa aumento do FSE e do VSE, que variam de maneira diretamente proporcional no início, enquanto a PIC não sobe novamente pela hipervolemia encefálica em progressão. Mais tarde, se o VSE continua aumentando, instala-se a HIC, que causa nova e secundária diminuição da PPE e do FSE.

$\mathrm{Na}$ fase inicial, o FSE é rápido (hiperemia), pois há PPE adequada em um leito capilar dilatado e de baixa resistência (inchaço hiperêmico). O consumo cerebral de oxigênio (cerebral metabolic rate of oxygen - $\mathrm{CMRO}_{2}$ ) é normal, a extração de oxigênio (diferença arteriovenosa de oxigênio - $\mathrm{DAVO}_{2}$ ) é baixa e estamos em perfusão de luxo. Mais tarde, se o VSE continua aumentando, a PIC começa a subir pela hipervolemia encefálica. Com a intensa vasodilatação, aumento do VSE, inchaço e HIC, associados à nova queda da PPE, o FSE diminui (inchaço oligoêmico). $\mathrm{O} \mathrm{CMRO}_{2}$ estará inicialmente normal, e a extração $\left(\mathrm{DAVO}_{2}\right)$, aumentada. Mais tarde, o $\mathrm{CMRO}_{2}$ cairá por insuficiência de oferta. Ocorreu, assim, uma hipoperfusão pós-hiperemia. . $^{2,7-711,22,32,41,42,48-50,71}$

\section{Hipoperfusão pós-hiperemia sob o ponto de vista bioquímico: segunda hipótese}

No terceiro tipo de isquemia, conforme relatado na introdução deste texto, uma situação intermediária, o fenômeno da hipoperfusão pós-hiperemia, ocorre após isquemias moderadas.

Nessa situação a acidose tecidual isquêmica associa-se à entrada de sódio na célula e à saída de potássio (falência anôxica da bomba iônica). Tal distúrbio permite a entrada celular de cálcio iônico que ativa fosfolipases que degradam os fosfolipídios das membranas celulares liberando ácidos graxos poli-insaturados, entre eles o ácido aracdônico que, sob ação da ciclooxigenase, dispara a cadeia das prostaglandinas. Em um de seus braços a cadeia oferece uma síntese intensa e de tromboxano A2 (TXA2), vasoconstritor e agregante plaquetário, desproporcionalmente grande em relação à síntese de prostaciclina. Assim, ocorrem vasoconstrição e agregação plaquetária que provocam uma diminuição da perfusão tecidual após uma hiperemia que vinha ocorrendo na reperfusão do leito vascular dilatado pela acidose. Ocorre, assim, também, uma hipoperfusão pós-hiperemia. $19,23,31,47,52,62,63,66,71$

\section{Acoplamento e desacoplamento}

Em uma conceituação simplista e inicial, ressalva-se que o aumento da atividade neuronal, localizada ou global, implica maior consumo de $\mathrm{O}_{2}$ e glicose, o que gera vasodilatação da área em atividade. De forma 
oposta, a diminuição da atividade elétrica e do consumo gera vasoconstrição. Esta é a variação fisiológica normal, ocorre quando a contratilidade da arteríola pré-capilar está íntegra (MARCC) e o sistema fluxo (oferta)/consumo está acoplado.

Se houver intensa acidose e isquemia tecidual iniciais, a vasodilatação se mantém intensa por tempo indeterminado a despeito de variações do consumo. O FSE e o VSE ficam aumentados inicialmente (hiperemia) e o FSE diminui com VSE alto em seguida (oligoemia). Existe desintegração na relação entre a oferta (fluxo) e o consumo, e o sistema está desacoplado.

\section{Relação das hipóteses fisiopatológicas com as ondas patológicas da HIC}

Ondas patológicas ocorrem no traçado da monitorização da PIC quando pequenos aumentos do VSE secundários à vasodilatação isquêmica adicional elevam de maneira desproporcional a PIC. Indicam que o compartimento intracraniano encontra-se na fase ascendente exponencial da curva de Langfitt. Nessa fase, a complacência do compartimento é quase nula e os exames de imagem demonstram apagamento dos sulcos, das cisternas e dos ventrículos, ou seja, extrusão do compartimento liquórico do crânio, que vinha compensando o aumento do VSE. As ondas patológicas retornam à linha de base espontaneamente porque a elevação da PIC causa uma ascensão reflexa da PAM (reflexo de Cushing), elevando a PPE e o FSE, reperfundindo o cérebro e revertendo a vasodilatação isquêmica. Assim, ocorre a diminuição do VSE e da PIC espontaneamente, ou seja, a redução do inchaço. Obviamente, cada onda patológica corresponde a um surto de isquemia reperfundida e suas consequências metabólicas são cumulativas. ${ }^{1,3,16-18,20,21,24,27,29,34,57,61}$

\section{Relação das hipóteses fisiopatológicas com o fenômeno da vulnerabilidade seletiva}

Mesmo em isquemias globais, o sofrimento do tecido encefálico é heterogêneo. Neurônios metabolicamente mais ativos sofrem antes dos menos ativos. Os primeiros provavelmente são, em geral, mais recentemente formados na escala ontofilogenética. Assim, um cérebro submetido à isquemia global é composto de distintos focos de sofrimento em diferentes idades evolutivas. Possivelmente, as alterações hemodinâmi- cas locais acompanham as alterações metabólicas, o que pode sugerir heterogeneidade de fluxo regional., ${ }^{6,58}$

\section{Relação da reperfusão pós-isquêmica com as isquemias de moderada intensidade}

Isquemias encefálicas globais completas e definitivas são raras na prática clínica e ocorrem apenas na parada cardiorrespiratória não revertida. Na prática clínica diária, geralmente ocorrem isquemias parciais de diferentes intensidades, cujo fluxo residual depende da persistência da PPE e do FSE regionais dentro dos próprios vasos em sofrimento ou por meio da circulação colateral. A lesão por reperfusão nada mais é do que um modelo laboratorial para se estudarem as repercussões bioquímicas das isquemias parciais.

A importância bioquímica das isquemias parciais ou reperfundidas baseia-se no fato de que um oferecimento de oxigênio muito insuficiente ou tardio, mas presente, libera a ciclooxigenase da cadeia das prostaglandinas, propiciando grande síntese de TXA2. Adicionalmente, o oxigênio molecular ganha um elétron e transforma-se em superóxido, um radical livre de oxigênio (ou elemento reativo) que, pela reação Haber-Weiss catalisada pelo ferro iônico, pode originar a hidroxila (OH-), fortemente oxidativa. Outras fontes de radicais livres incluem a degradação da hemoglobina, o metabolismo da adenosina pela xantina-oxidase, o metabolismo do óxido nítrico e a degradação das catecolaminas. $\mathrm{O}$ fenômeno se intensifica pela impossibilidade de captação dos elementos reativos pelas mitocôndrias sob anaerobiose.

Esses fatos, obviamente, não indicam que não se deva reoxigenar o tecido, mas indicam que neuroprotetores bioquímicos devem ser pesquisados. Esses fenômenos também são a base do que se chama inflamação do tecido isquêmico.

\section{Fenômeno da parada circulatória intracraniana}

Após estabelecimento de grave HIC a pressão venosa de drenagem assume o valor da PIC e da PAM. Embora não haja especificamente uma compressão obstrutiva do sistema venoso de capacitância, a pressão venosa aumentada, equiparando-se à pressão arterial de entrada, neutraliza o gradiente pressórico arteriovenoso, e o fluxo sanguíneo no sistema vascular tubular, dependente desse gradiente, para. ${ }^{70}$ 


\section{Implicações terapêuticas}

A reperfusão tecidual deve ser precoce para que alterações metabólicas sejam minimizadas. Ondas patológicas ao traçado de monitorização da PIC já indicam tratamento agressivo da HIC. O equilíbrio da relação entre a oferta e o consumo deve ser obtido pela normalização do primeiro ou pela diminuição do segundo, reduzindo assim a acidose e suas consequências desencadeadoras das cascatas vasodilatadora e bioquímica. 5,60

Situações de isquemias reperfundidas ou parciais ocorrem após a ressuscitação de um paciente que apresentou parada cardiorrespiratória ou após a remoção de um hematoma intracraniano de qualquer etiologia, ou como consequência de repetidos surtos de HIC que podem ocorrer dentro da UTI. A precocidade da ação médica gera a principal neuroproteção, por diminuir a acidose tecidual. ${ }^{71}$

\section{Referências}

1. Auer LM, Sayama I. Intracranial pressure oscillation (B Waves) caused by oscillation in cerebrovascular volume. Acta Neurochir (Wien). 1983;68:93-100.

2. Berman IR, Ducker TB, Simmons RL. The effects of increased intracranial presure uppon the oxigenation of blood in dogs. J Neurosurg. 1969;30:532-6.

3. Brock M, Winkelmuller W, Poll W, Markakis E, Dietz $\mathrm{H}$. Measurements of brain tissue pressures. Lancet. 1972;2:595-6.

4. Bruce DA, Langfitt TW, Miller JD, Schutz H, Valpachti MP, Staneck A, et al. Regional cerebral blood flow, intracranial pressure and brain metabolism in comatose patients. J Neurosurg. 1973;38:131-44.

5. Clark RM, Lapra NF, Halsey JH. Method for measuring brain tissue pressure: response to alterations in $\mathrm{PCO} 2$, systemic blood pressure and middle cerebral artery occlusion. $J$ Neurosurg. 1975;43:1-8.

6. Crockard A, lannotti F, Hunstock AT, Smith RD, Harris RJ, Symon L. Cerebral blood flow and edema following carotid occlusion in the gerbil. Stroke. 1980;11:494-8.

7. Cruz J. Monitorização hemodinâmica e metabólica cerebral em humanos. Arq Bras Neurocir. 1992;1:209-15.

8. Cruz J, Gennarelli TA, Alves WM. Continuous monitoring of cerebral hemodynamic reserve in acute brain injury: relationship to changes in brain swelling. $J$ Trauma. 1992;32:629-35.

9. Cruz J, Miner ME, Allen SJ, Alves WM, Gennarelli TA. Continuous monitoring of cerebral oxygenation in acute brain injury: assessment of cerebral hemodynamic reserve. Neurosurgery. 1991;29:743-9.

10. Denny-Brown D, Meyer J. The cerebral collateral circulation. II. Production of cerebral infarction by ischemic anoxia and its reversibility in early stages. Neurology. 1957;7:567-79.

11. Enevoldsen EM, Cold G, Jensen FT, Malmros R. Dynamic changes in regional $\mathrm{CBF}$, intracranial pressure, CSF pH, and lactate levels during the acute phase of head injury. $J$ Neurosurg. 1976;44:191-214.

12. Enevoldesen EM, Jensen FT. Compartmental analysis of regional cerebral blood flow in patients with acute severe head injury. J Neurosurg. 1977;47:699-712.
13. Fieschi C, Battistini N, Beduschi A, Boselli L, Rossanda M. Regional cerebral flood flow and intravascular pressure in the acute head injuries. J Neurol Neurosurg Psychiatry. 1974;37:1378-88.

14. Fischer EG. Impaired perfusion following cerebrovascular stasis. Arch Neurol. 1973;29:361-6.

15. Fischer EG, Arnes A III, Lorenzo AV. Cerebral blood flow immediately following brief circulatory stasis. Stroke. 1979;10:423-7.

16. Fitch W, McDowall EG. Systemic vascular responses to increased intracranial pressure: 1 . Effects of progressive intracranial epidural balloon expansion on intracranial pressure and systemic circulation. J Neurol Neurosurg Psychiatry. 40:833-42.

17. Fitch W, McDowall DG, Keaney MP, Pickerodt VWA. Systemic vascular responses to increased intracranial pressure: 2. The "Cushing" response in the presence of intracranial space-occupying lesions: systemic and cerebral haemodinamic studies in the dog and the baboon. J Neurol Neurosurg Psychiatry. 1977; 40:843-52.

18. Grady PA, Blaumanis OR. Cerebral venous blood gas tensions in elevation of intracranial pressure. Stroke. 1986;17:946-52.

19. Grahan DI, Adams JH. Ischemic brain damage in head injuries. Lancet. 1971;1:265-6.

20. Gray WJ, Rosner MJ. Pressure-volume index as a function of cerebral perfusion pressure. Parte. I. The effects of cerebral perfusion pressures changes and anesthesic. J Neurosurg. 1987;67:369-76.

21. Gray WJ, Rosner MJ. Pressure-volume index as a function of cerebral perfusion pressure. Part 2. The effects of low cerebral perfusion pressure and autoregulation. $J$ Neurosurg. 1987;67:377-80.

22. Grubb Jr. RL, Raichle ME, Phelps ME, Patcheson PA. Effects of increased intracranial pressure on cerebral blood volume, blood flow and oxygen utilization in monkeys. J Neurosurg. 1975;43:385-98.

23. Gursoy-Ozdemir L, Gan A, Dalkara T. Reperfusion induced oxidative/nitrosative injury to neurovascular units after local cerebral ischemia. Stroke. 2004;35:1449-53.

24. Hamer J, Albert F, Hoyer S, Wiedemann K. Influence of systemic and cerebrovascular factors on cerebrospinal fluid pulse waves. J Neurosurg. 1977;46:36-45.

25. Harper AM. Auto-regulation of cerebral blood flow: influence of the arterial blood pressure on the blood flow through the cerebral cortex. J Neurol Neurosurg Psychiatry. 1966;29:398-403

26. Hedges TF, Weinstein JD. Cerebrovascular responses to increased intracranial pressure. J Neurosurg. 1964;21:292-7.

27. Hekmatpanah J. The sequence of alterations in the vital signs during acute experimental increased intracranial pressure. J Neurosurg. 1970;32:16-20.

28. Hekmatpanah J. Cerebral circulation and perfusion in experimental increased intracranial pressure. J Neurosurg. 1970;32:21-9.

29. lannotti F, Hoff JT, Schielke GP. Brain tissue pressure: physiological observations in anesthetized cats. J Neurosurg. 1984;60:1219-25.

30. lannotti F, Hoff JT, Schielke GP. Brain tissue pressure in focal cerebral ischemia. J Neurosurg. 1985;62:83-9.

31. Ikeda $Y$, Long DM. The molecular basis of brain injury and brain edema: the role of oxygen free radicals. Neurosurgery. 1990;27:1-11.

32. Johnston IH, Rowan JO. Raised intracranial pressure and cerebral blood flow. 3. Venous outflow tract pressures and vascular resistances in experimental intracranial hypertension. J Neurol Neurosurg Psychiatry. 1974;37:392402. 
33. Johnston IH, Rowan JO, Harper AM, Jennet WB. Raised intracranial pressure and cerebral blood flow. 1. Cisterna magna infusion on primates. J Neurol Neurosurg Psychiatry. 1972;35:285-96.

34. Johnston IH, Rowan JO, Park DM, Rennie MJ. Raised intracranial pressure and cerebral blood flow. 5. Effects of episodic intracranial pressure waves in primates. J Neurol Neurosurg Psychiatry. 1975;38:1076-82.

35. Kato Y, Auer LM. Cerebrovascular response to elevation of ventricular pressure. Acta Neurochir. 1989;98:184-8.

36. Kjallqvist $\mathrm{A}$, Lundberg $\mathrm{N}$, Ponten $\mathrm{U}$. espiratory and cardiovascular changes during rapid spontaneous variations of ventricular fluid pressure in patients with intracranial hypertension. Acta Neurol Scand. 1964;40:291-317.

37. Langfitt TW, Kassell NF. Acute brain swelling in neurosurgical patients. J Neurosurg. 1966;24:975-83.

38. Langfitt TW, Kassell NF, Weinstein JD. Cerebral blood flow with intracranial hypertension. Neurology (Mineap). 1965;15:761-73.

39. Langfitt TW, Weinstein JD, Kasse NF. Cerebral vasomotor paralysis produced by intracranial hypertension. Neurology. 1965;15:622-41.

40. Langfitt TW, Weinstein JD, Kassel NF, Gagliard, LJ, Shapiro HM. Compression of cerebral vessels by intracranial hypertension. I. Dural sinus pressures. Acta Neurochir (Wien). 1966;15:212-22.

41. Lassen NA. The luxury-perfusion syndrome and its possible relation to acute metabolic acidosis localized within the brain. Lancet. 1966;2:1113-5.

42. Lenzi G, Franckowiak RSJ, Jones T. Cerebral oxygen metabolism and blood flow in human cerebral ischemic infarction. J Cereb Blood Flow Metab. 1982;2:321-35.

43. Levy DE, Pike RL, Uitert RL. Delayed dissociation of cerebral blood flow and metabolism following stroke in gerbils. Neurology. 1978;28:378-9.

44. Levy DE, Van Uitert RL, Pike CL. Delayed post-ischemic hypoperfusion: a potentially damaging consequence of stroke. Neurology. 1979; 29:1245-52.

45. Löfgren J, Von Essen C, Zwetnow NN. The pressure volume curve of the cerebropinal fluid space in dogs. Acta Neurol Scand. 1973;49:557-74.

46. Lowell HM, Bloor BM. The effect of increased intracranial pressure on cerebrovascular hemodynamics. J Neurosurg. 1971:34:760-9.

47. Maier CM, Hsieh L, Crandall T, Narasimhan P, Chan PH. A new approach for the investigation of reperfusion related brain injury. Bioch Soc Transac. 2006;34:1366-9.

48. Marshall LF, Durity F, Lounsbury R, Graham DI, Welsh F, Langfitt TW. Experimental cerebral oligoemia and ischemia produced by intracranial hypertension. Part 1 . Pathophysiology, electroencephalography, cerebral blood flow, blood-brain barrier, and neurological function. J Neurosurg. 1975;43:308-17.

49. Marschall LF, Graham DI, Durity F, Lounsbury R, Welsh F, Langfitt TW. Experimental cerebral oligoemia and ischemia produced by intracranial hypertension. Part. 2. Brain morphology. J Neurosurg. 1975;43:318-22.

50. Marshall LF, Welsh F, Durity F, Lounsbury R, Graham DI, Langfitt TW. Experimental cerebral oligoemia and ischemia produced by intracranial hypertension. Part. 3. Brain energy metabolism. J Neurosurg. 1975;43:323-8.

51. Marshall WJS, Jackson ILF, Langfitt TW. Brain swelling caused by trauma and arterial hypertension: hemodynamic aspects. Arch Neurol. 1969;21:545-53.

52. Melgar MA, Rafols J, Groos D, Diaz FG. Postischemic reperfusion: ultrastructural blood-brain barrier and hemodinamic correlative changes in a awake model of transient forebrain isquemia. Neurosurgery. 2005;56:571-82.

53. Miller JD, Garibi J, North JB, Teasdale GM. Effects of increased arterial pressure on blood flow in the damaged brain. J Neurol Neurosurg Psychiatry. 1975;38:657-65.
54. Miller JD, Stanock A, Langfitt TW. Concepts of cerebral perfusion pressure and vascular compressions during intracranial hypertension. Prog Brain Res. 1971;35: 411-32.

55. Miller JD, Stanock A, Langfitt TW. Cerebral blood flow regulations during experimental brain compression. J Neurosurg. 1973;39:186-96.

56. Nakagawa Y, Yamamoto L, Meyer E, Hodge CP, Feindel W. Effects of hypercapnia on enhancement of decreased perfusion flow in non-infarcted brain tissue. Stroke. 1981; 12:85-92.

57. O'Brist WD, Langfitt TW, Jaggi JL, Cruz J, Gennarelli TA. Cerebral blood flow and metabolism in comatose patients with acute head injury. Relationship to intracranial hypertension. J Neurosurg. 1984;61:241-53.

58. Olsen TS, Larsen B, Herning M, Skriver EB, Lassen NA. Blood flow and vascular reactivity in collaterally perfured brain tissue: evidence of an ischemic penumbra in patients with acute stroke. Stroke. 1983;14:332-42.

59. Olsen TS, Larsen B, Skriver BE, Herning M, Enevoldsen $\mathrm{E}$, Lassen NA. Focal cerebral hyperemia in acute stroke. Incidence, pathophysiology and clinical significance. Stroke. 1981:12:589-607.

60. Reulen HJ, Kreysch HG. Measurement of brain tissue pressure in cold induced cerebral edema. Acta Neurochir (Wien). 1973;29:29-40.

61. Risberg J, Lundberg N, Ingvar DM. Regional cerebral blood volume during acute transient rises of the intracranial pressure (plateau waves). J Neurosurg. 1969;31:303-10.

62. Ropper AH, Shafran B. Brain edema after stroke. Clinical syndrome and intracranial pressure. Arch Neurol. 1984;41:26-9.

63. Schutz H, Silverstein PR, Vapalahti M, Bruce DA, Mela L, Langfitt TW. Brain mitochondrial function after ischemia and hypoxia. 1. Ischemia produced by increased intracranial pressure. Arch Neurol. 1973;29:408-16.

64. Smith DR, Jacobson J, Kobrine AL, Rizzoli HV. Regional cerebral blood flow with intracranial mass lesions. Part I. Local alterations in cerebral blood flow. Surg Neurol. 1977;7:233-7.

65. Smith DR, Jacobson J, Kobrine Al, Rizzoli HV. Regional cerebral blood flow with intracranial mass lesions. Part II. Autoregulation in localized mass lesion. Surg Neurol. 1977;7:238-40.

66. Stavale M. Fisiopatologia básica da isquemia encefálica aguda. In: Stávale M (Ed.). Bases da terapia intensiva neurológica. São Paulo: Editora Santos, 1996. p.13-24.

67. Symon L, Branstson NM, Strong AJ. Autoregulation in acute focal ischemia. An experimental study. Stroke. 1981;7: 547-54.

68. Waltz AG. Effects of PaCO2 on blood flow and microvascular of ischemic and non ischemic cerebral cortex. Stroke. 1970;2:27-37.

69. Weinstein JD, Langfitt TW, Bruno CA, Zaren HA, Jackson JLF. Experimental study of patterns of brain distortion and ischemia produced by an intracranial mass. J Neurosurg. 1968;28:513-21.

70. Yada K, Nakagawa Y, Tsuru M. Circulatory disturbance of the venous system during experimental intracranial hypertension. J Neurosurg. 1973;39:723-9.

71. Zwetnow N, Kjällquist A, Siesjo BK. Cerebral blood flow during intracranial hypertension in relation to tissue hipoxia and to acidosis in cerebral extracellular fluids. Prog Brain Res. 1968;30:87-92.

Endereço para correspondência

Marcos Augusto Stávale Joaquim

Alameda Campinas, $1.360,16^{\circ}$ andar

01404-002 - São Paulo, SP

Email:marcos.stavale@terra.com.br 